\section{Perfil de produção do exame citopatológico para controle do câncer do colo do útero em Minas Gerais, Brasil, em 2002}

\author{
Pap smear screening for the control of cervical \\ cancer in Minas Gerais State, Brazil, 2002
}

\author{
I Instituto Nacional de \\ Câncer, Ministério da Saúde, \\ Rio de Janeiro, Brasil. \\ 2 Instituto Alberto Luis \\ Coimbra de Pós-graduação \\ em Engenharia, Universidade \\ Federal do Rio de Janeiro, \\ Rio de Janeiro, Brasil. \\ Correspondência \\ T. M. P. Feitosa \\ Instituto Nacional de Câncer, \\ Ministério da Saúde. \\ Rua do Rezende 195, sala 205, \\ Rio de Janeiro, $R J$ \\ 20231-091, Brasil. \\ tfeitosa@inca.gov.br
}

\begin{abstract}
This article analyzes the implementation of Pap smears by the Cervical Cancer Control Program in 2002 in women 25-59 years of age residing in 850 municipalities (counties) in Minas Gerais State, Brazil. Cluster analysis was performed to classify municipalities by: number of Pap smears performed; test findings; and adequacy of slides. The variable "satisfactory slide, but limited by" was the principal factor for classifying municipalities into 5 clusters. Spatial representation of clusters indicated a more critical situation in the North, Jequitinhonha, Vale do Mucuri, and Vale do Rio Doce "meso-regions" of Minas Gerais and identified operational problems resulting from inadequate collection of material, with more "desiccated" slides observed in the North of the State and more slides with "absent endocervical cells" in the Center and South. The methodology allowed identifying clusters of municipalities with problems in the screening process, related to quality of sampling and fixing and reading of slides.
\end{abstract}

Uterine Cervical Neoplasms; Mass Screening; Cytological Techniques
Tereza Maria Piccinini Feitosa 1,2 Rosimary Terezinha de Almeida 2

\section{Introdução}

A incidência do câncer de colo do útero em um país é conseqüência da exposição das mulheres a fatores de risco e da efetividade de um programa de rastreamento (screening). Sendo o rastreamento a identificação da doença ainda não reconhecida, por meio de exames diagnósticos ou clínicos, seu sucesso é dependente da existência de um número suficiente de profissionais de saúde treinados para realizar os exames de detecção precoce e confirmação diagnóstica, bem como o tratamento e acompanhamento da mulher 1,2 .

O exame citopatológico do colo do útero (Papanicolaou), recomendado pela Organização Mundial da Saúde para o rastreamento, tem se mostrado efetivo em reduzir a incidência e a mortalidade da doença, quando disponibilizado em países com programas estruturados 3 . No Brasil, apesar de introduzido na rede pública de serviços aproximadamente há 25 anos, integrando programas regionais bem sucedidos 4 e fazer parte do Sistema de Informações Ambulatoriais do Sistema Único de Saúde (SIA/SUS) 5 desde 1994, o exame (Papanicolaou) ainda é oferecido às mulheres de forma oportunista, quando estas comparecem à unidade de saúde para atendimento, principalmente para cuidados materno-infantil 6 . Pesquisa realizada pelo Ministério da Saúde em 15 capitais brasileiras e Distrito Federal, de 2002 a 2003, mostrou uma variação de 73,4\% (João Pessoa, Paraíba) a 92,9\% (Vitória, 
Espírito Santo) de mulheres com idade igual ou superior a 15 anos, relatando ter feito pelo menos um exame nos últimos três anos 7 .

Cabe ressaltar que a taxa de mortalidade por câncer do colo do útero teve um crescimento de $30 \%$ em 23 anos, passando de 3,44 casos por 100 mil mulheres em 1979, para 5,03 em 2002 7,8,9. Para 2006, estima-se que a incidência de câncer do colo do útero seja de 19.260 casos novos, com um risco estimado de vinte casos a cada $100 \mathrm{mil}$ mulheres 10. É importante acrescentar, com base em registros hospitalares de câncer, que 50\% das mulheres portadoras da doença foram diagnosticadas em estádios III e IV 7,8,9. Isso, provavelmente reflete a dificuldade de diagnóstico precoce, a falta de informação e a dificuldade de acesso da mulher à rede de saúde.

O Ministério da Saúde, por intermédio do Instituto Nacional de Câncer (INCA), em parceria com as Secretarias de Saúde, desenvolve o Viva Mulher - Programa Nacional de Controle do Câncer do Colo do Útero e de Mama, direcionando o rastreamento do câncer do colo do útero para as mulheres na faixa etária de 25 a 59 anos. Com o objetivo de reduzir a mortalidade por esse tipo de câncer, são estratégias do Viva Mulher: disponibilizar o exame (Papanicolaou); oferecer o tratamento adequado da doença e de suas lesões precursoras em $100 \%$ dos casos; e monitorar a qualidade do atendimento à mulher, em todas as suas etapas (prevenção e detecção precoce, tratamento e reabilitação) ${ }^{6,8}$. Em 1998 foi realizada a primeira fase de intensificação do Viva Mulher, dirigida às mulheres de 35 a 49 anos que nunca realizaram o exame citopatológico do colo do útero. Em 2002, ocorreu uma segunda fase de intensificação, voltada para a mesma faixa etária, tendo como foco as mulheres que nunca realizaram o exame (Papanicolaou) ou que o fizeram há três anos ou mais. As duas fases de intensificação do programa foram caracterizadas por uma grande mobilização nacional das mulheres, por meio de uma campanha de publicidade, e pelo oferecimento facilitado do exame nos serviços de saúde dos municípios, em um período de aproximadamente três meses 8 .

Uma vez que as ações realizadas são essencialmente em nível municipal, devem ser consideradas a situação socioeconômica e as desigualdades existentes, quando se formula uma política de controle do câncer 11 . Nesse sentido, o nível educacional das mulheres pode influenciar sobremaneira o acesso à informação e a incorporação de hábitos e comportamentos favoráveis à prevenção da doença, tais como a realização do exame (Papanicolaou) 7,12. Assim, pode-se considerar que o desempenho do Programa seja influenciado por três grandes vertentes: (1) capaci- dade de atingir seu objetivo em cada município, por meio de suas estratégias; (2) capacidade operacional da rede de serviços de saúde na coleta adequada de material que revele o câncer ou sua lesão precursora, e na interpretação técnica do material pelo laboratório de citopatologia; e (3) nível sócio-econômico das mulheres 8,13,14 .

O objetivo deste trabalho é estudar o perfil de produção do exame citopatológico (Papanicolaou) das mulheres na faixa etária de 25 a 59 anos, residentes em 850 municípios do Estado de Minas Gerais, Brasil, durante o ano de 2002, registrado pelo Departamento de Informação e Informática do SUS (DATASUS), considerando as três vertentes acima mencionadas 15 .

\section{Métodos}

Foram analisados 981.316 exames citopatológicos do colo do útero (procedimento 12.011.01-0, do SIA/SUS) de mulheres residentes em $850 \mathrm{mu}$ nicípios, na faixa etária de 25 a 59 anos, registrados no ano de 2002 no Sistema de Informações do Câncer da Mulher (SISCAM-SIA/SUS) 15. Esse sistema foi criado para monitorar o processo de rastreamento populacional e o tratamento das lesões precursoras do câncer do colo do útero 8 .

As variáveis usadas no estudo foram: (1) razão de exames realizados na população alvo; (2) percentual de mulheres que informaram não ter realizado o exame anteriormente; (3) percentual de exames apresentando efeito citopático compatível com HPV (vírus do papiloma humano); (4) percentual de exames apresentando Neoplasia Intra-epitelial Cervical - NIC I (displasia leve); (5) percentual de exames apresentando NIC II (displasia moderada); (6) percentual de exames apresentando NIC III (displasia acentuada); (7) percentual de exames apresentando carcinoma escamoso invasivo; (8) percentual de lâminas consideradas com adequabilidade "satisfatória, mas limitada por"; (9) percentual de lâminas consideradas com adequabilidade "satisfatória, mas limitada por ausência de células endocervicais" (subcategoria da variável 8); (10) percentual de lâminas consideradas com adequabilidade "satisfatória, mas limitada por dessecamento" (também subcategoria de 8); (11) percentual de lâminas consideradas com adequabilidade "insatisfatória"; e (12) taxa de não-alfabetizadas no ano 2000 (obtida da base demográfica do Instituto Brasileiro de Geografia e Estatística - IBGE) 6,14,15,16.

A escolha dessas variáveis considerou as três vertentes que influenciam o Viva Mulher. A primeira, relativa ao cumprimento do objetivo e estratégias do programa, foi representada pelas 
variáveis 1 e 2, referentes ao acesso das mulheres ao rastreamento, e pelas variáveis 3 a 7, relativas à ocorrência de lâminas com câncer do colo do útero e suas lesões precursoras em Minas Gerais. A segunda vertente, referente à capacidade operacional da rede de serviços de saúde, foi representada quanto à coleta adequada do material que revele o câncer ou sua lesão precursora, pelas variáveis 8 a 11 e, quanto à interpretação técnica do material pelo laboratório de citopatologia, pelas variáveis 3 a 7. A terceira vertente, referente ao nível sócio-econômico das mulheres foi representada pela variável 12, pois o baixo nível de escolaridade pode influenciar na adoção de atitudes de autocuidado em saúde 12.

No intuito de classificar os municípios que apresentassem características similares quanto à realização do exame citopatológico (Papanicoloau) e identificar pontos críticos do programa, realizou-se o estudo em três etapas.

$\mathrm{Na}$ primeira, fez-se uma análise de agrupamento (cluster analysis) usando o programa SPSS versão 6.0 (SPSS Inc., Chicago, Estados Unidos). As variáveis utilizadas foram: 1 (razão de exames realizados na população); 3 a 7 (tipos de lesão encontrada nas lâminas); 8 e 11 (coleta adequada do material que revele o câncer ou sua lesão precursora). Entre os métodos de agregação hierárquica, foi escolhido o método de Ward por permitir uma melhor visualização dos grupos formados, sendo realizada uma análise exploratória buscando identificar as características desses grupos. Uma vez identificado um número de grupos com características distintas, utilizou-se o método K-means no sentido de confirmar os grupos identificados 17,18,19,20. Foram excluídos da análise, por não apresentarem produção de exames, os Municípios de Setubinha, Estrela Dalva e Santo Antônio do Aventureiro, do Estado de Minas Gerais.

Na segunda etapa, fez-se a descrição dos grupos obtidos no sentido de caracterizá-los quanto à realização do exame citopatológico, tendo como base a localização dos municípios de cada grupo nas mesorregiões.

Na terceira, fez-se a representação espacial dos grupos de municípios, sendo superpostas as variáveis não utilizadas na análise de agrupamento: 2, 9, 10 e 12. Para tanto, utilizou-se o programa TabWin versão 3.0 e os mapas distribuídos pelo DATASUS, a partir dos CD-ROMs do IBGE, na escala de 1:250.000, Malha Municipal Digital do Brasil - Situação em 2001 - Volume 1 - Projeção Geográfica.

\section{Resultados}

A primeira etapa (análise de agrupamento) iniciou-se pela análise exploratória das variáveis usadas para a classificação dos municípios, mostrando que o Estado de Minas Gerais teve os seguintes valores para o 1ㅇ, 2o e 3 o quartis, respectivamente:

- Razão de exames realizados na população alvo $(0,21 ; 0,27$ e 0,34$)$;

- Percentual de exames apresentando efeito citopático compatível com HPV (0; 0,21 e 0,52);

- Percentual de exames apresentando NIC I (0; 0,38 e 0,76);

- Percentual de exames apresentando NIC II (0; 0 e 0,25$)$;

- Percentual de exames apresentando NIC III (0; 0 e 0,19)

- Percentual de exames apresentando carcinoma escamoso invasivo (0 para os três quartis);

- Percentual de lâminas consideradas com adequabilidade "satisfatória, mas limitada por" (17,24; 28,78 e 44,60);

- Percentual de lâminas consideradas com adequabilidade "insatisfatória” (0; 0,49 e 1,22).

A análise de agrupamento identificou cinco grupos de municípios para os dois métodos utilizados, Ward e K-means. A variável percentual de lâminas consideradas com adequabilidade "satisfatória, mas limitada por" foi a que melhor discriminou os grupos, conforme a Figura 1, elaborada no método $K$-means, com variação da mediana entre os grupos de $8,90 \%$ a $70,02 \%$. Quando comparadas as medianas dos grupos com a mediana do estado $(28,78 \%)$ tem-se que os grupos 1 e 2 estão abaixo da linha representativa da mediana no estado e os grupos 3, 4 e 5 acima, estando, porém, os grupos 2 e 3 mais próximos da mediana estadual.

As demais variáveis pouco discriminaram os grupos, sendo que a maior variação ocorreu para o percentual de exames apresentando efeito citopático compatível com HPV (0 a 0,24$)$; o percentual de exames apresentando NIC I $(0,33$ a 0,48$)$; e o de lâminas consideradas com adequabilidade “insatisfatória” (0,14 a 0,66).

Assim, os grupos identificados caracterizaram os municípios em termos da qualidade na coleta e preservação do material, sendo que o grupo 1 estaria representado pelos municípios com melhor qualidade; os grupos 2 e 3, com qualidade intermediária; e grupos 4 e 5 , com pior qualidade.

Na segunda etapa, tomando-se como base a localização dos municípios de cada grupo nas mesorregiões, observou-se que:

- Grupo 1, com 70\% dos municípios localizados em seis mesorregiões, concentrou-se do centro para o sul do Estado; 
Figura 1

Distribuição percentual de lâminas consideradas com adequabilidade "satisfatória, mas limitada por", para os cinco grupos de municípios de Minas Gerais, Brasil, em 2002.

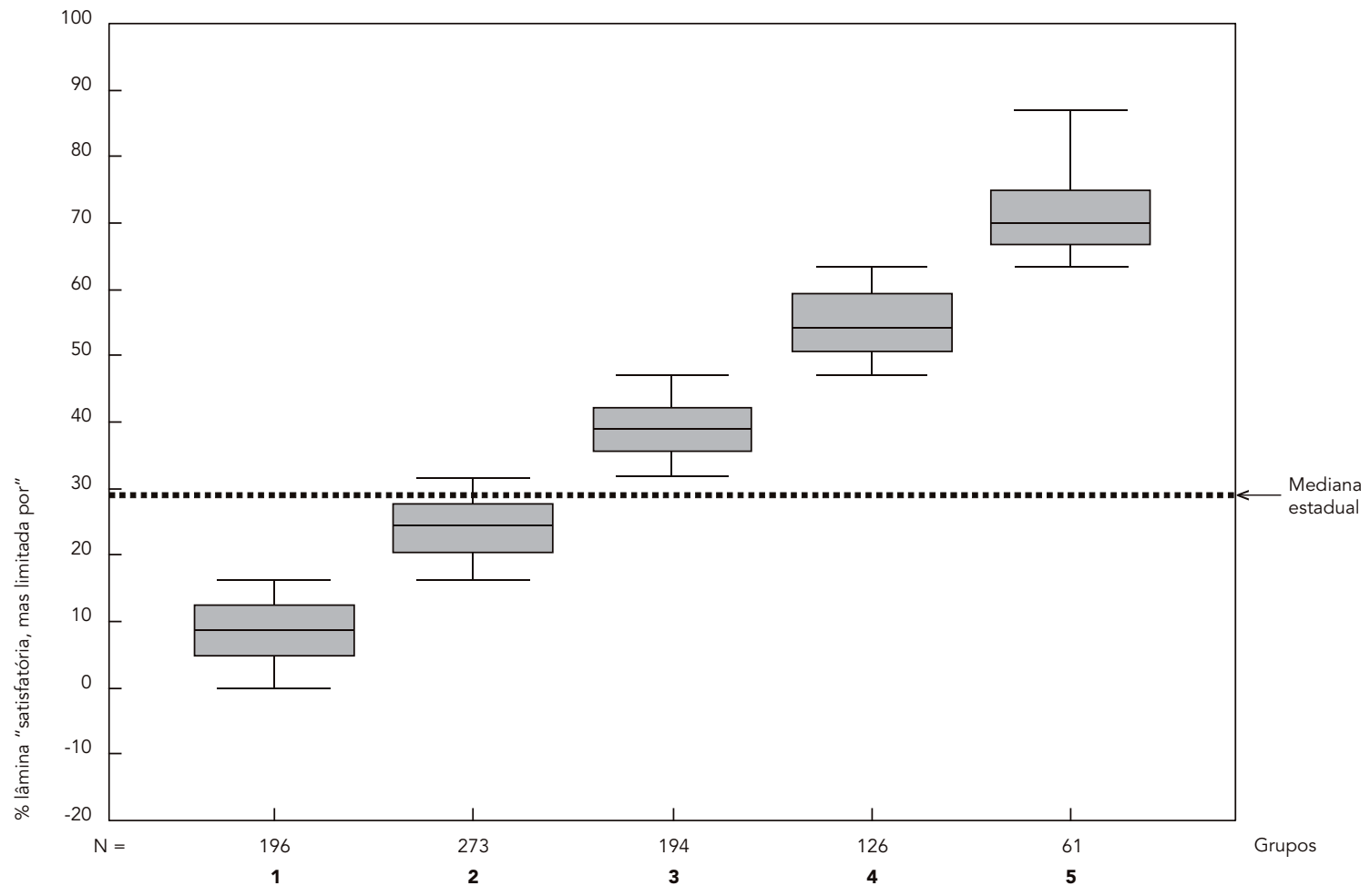

$\mathrm{N}$ : número de municípios por grupo.

- Grupo 2, com 70\% dos municípios dispersando-se em quatro mesorregiões, concentrou $26 \%$ na sul/sudoeste, com tendência para localizações mais ao norte que o grupo 1 ;

- Grupo 3, com 70\% dos municípios em cinco mesorregiões, concentrou-se na sul/sudoeste (20,6\%), ampliando-se ao centro e norte do Estado;

- Grupo 4, com 70\% dos municípios concentrando-se em cinco mesorregiões, reduziu a proporção de municípios ao sul e ampliou-a do centro para concentrar-se na mesorregião norte (23\%);

- Grupo 5 teve $80 \%$ dos municípios localizados em quatro mesorregiões, predominantemente ao norte (mesorregiões norte com $27,8 \%$ e Jequitinhonha com 23\%) e leste do Estado.

Na terceira etapa, a representação espacial dos grupos no mapa do Estado de Minas Gerais (Figura 2) identificou uma maior concentração de municípios dos grupos 4 e 5 ao norte, representados nos tons mais escuros simbolizando os resultados referentes aos maiores percentuais de lâminas consideradas com adequabilidade "satisfatória, mas limitada por”. Essa concentração se distribui em três faixas: a primeira numa larga faixa margeando a fronteira com o Estado da Bahia, em sentido leste-oeste, e as duas outras no sentido norte-sul, uma descendo pelo centro do mapa e a outra margeando a região fronteiriça com os Estados do Espírito Santo e Rio de Janeiro. É possível ainda observar, no restante do estado, pequenas concentrações desses grupos. Os tons mais claros, menores percentuais de lâminas consideradas com adequabilidade "satisfatória, mas limitada por", referentes aos grupos 1 e 2, se concentram do centro para o sul do mapa. No entanto, zonas com menores percentuais se mesclam com aquelas de maiores percentuais, como por exemplo, na região norte, onde os tons mais claros passam pelo centro da região, em sentido leste-oeste. Porém, na mesorregião Jequitinhonha foi nítida a separação em duas faixas: uma escura ao norte e uma clara ao sul. 


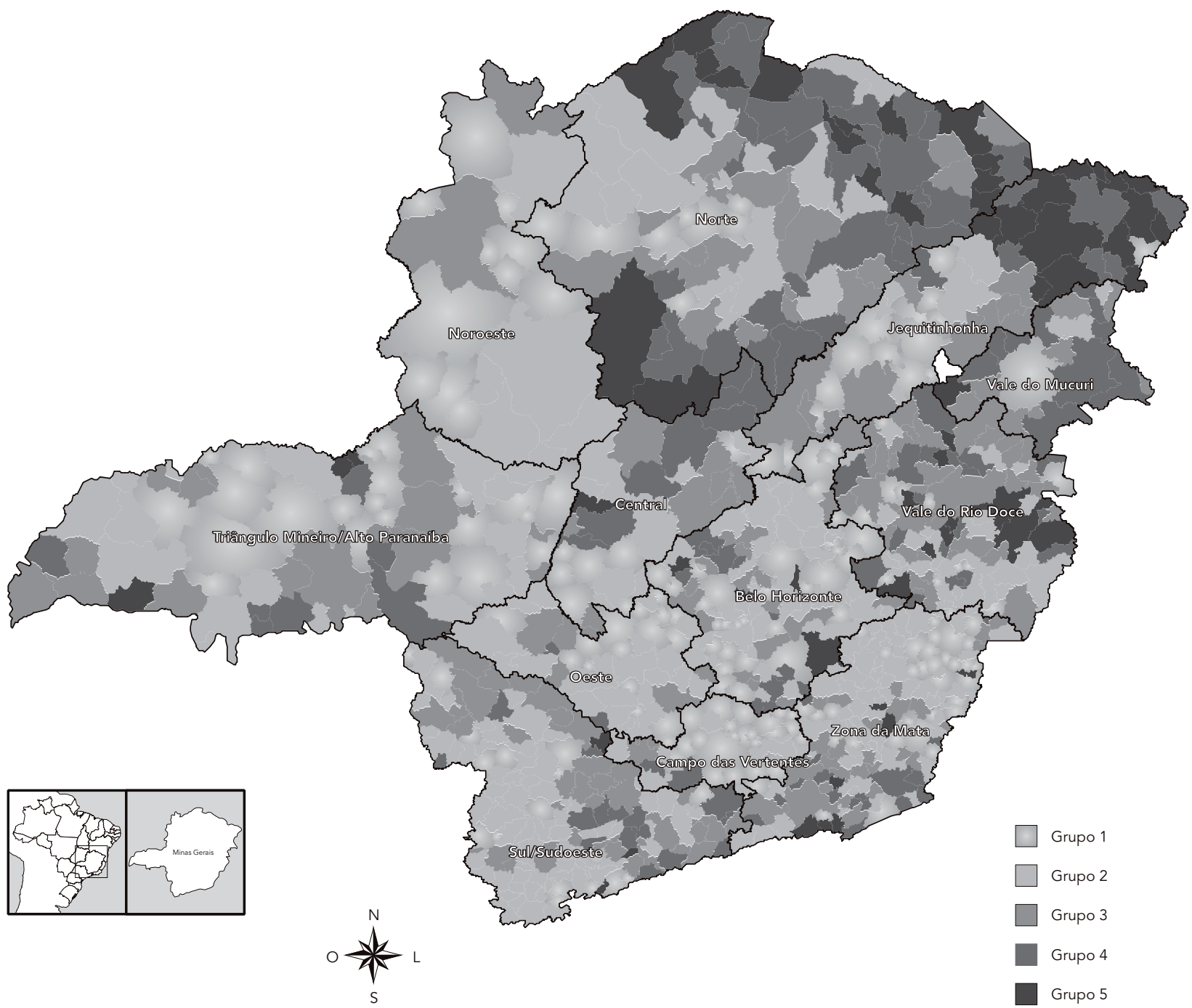

Uma vez que a variável percentual de lâminas consideradas com adequabilidade "satisfatória, mas limitada por" foi a mais significativa na discriminação dos grupos, foram analisadas as suas sete subcategorias, no sentido de identificar os pontos críticos dos programas municipais quanto à capacidade operacional da coleta de material para o exame (Papanicolaou). A representação espacial das duas subcategorias mais freqüentes - "ausência de células endocervicais" e "dessecamento" - sobre o mapa representativo dos grupos, permitiu a visualização do desempenho do Viva Mulher quanto à segunda vertente, ou seja, das condições operacionais do mesmo. Na Figura 3a, observa-se que os maiores percentuais da subcategoria "ausência de células endocervicais” (círculos proporcionais) concentraram-se do centro para o sul do Estado, enquanto que, na Figura 3b, as maiores proporções da subcategoria "dessecamento" concentraram-se ao norte.

A Figura 4a mostra a representação espacial da proporção de mulheres que informaram não ter realizado o exame citopatológico anteriormente $(67,8 \%$ responderam à pergunta), possibilitando observar o desempenho do programa quanto à primeira vertente (capacidade de atingir 
Figura 3

Representação espacial dos cinco grupos de municípios de Minas Gerais, Brasil, em 2002, com círculos proporcionais indicando: distribuições percentuais das lâminas consideradas com adequabilidade "satisfatória, mas limitada por ausência de células endocervicais" (3a); e distribuições percentuais das lâminas consideradas com adequabilidade "satisfatória, mas limitada por dessecamento" (3b).

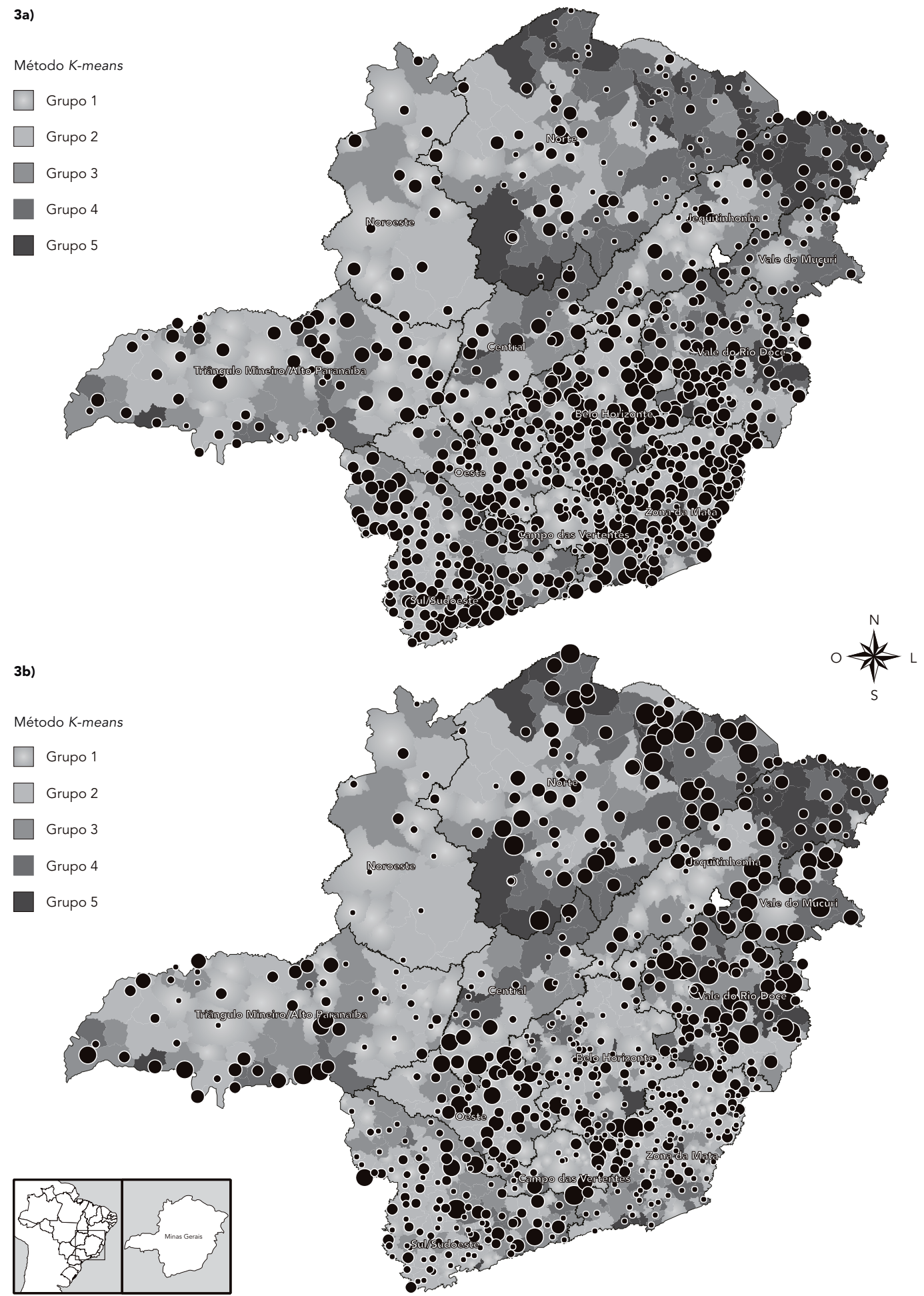


Representação espacial dos cinco grupos de municípios de Minas Gerais, Brasil, com círculos proporcionais indicando: distribuição percentual das mulheres que informaram, no ano de 2002, não ter realizado o exame citopatológico anteriormente (4a); e proporção de mulheres não-alfabetizadas em Minas Gerais, Brasil, ano 2000, por município (4b).

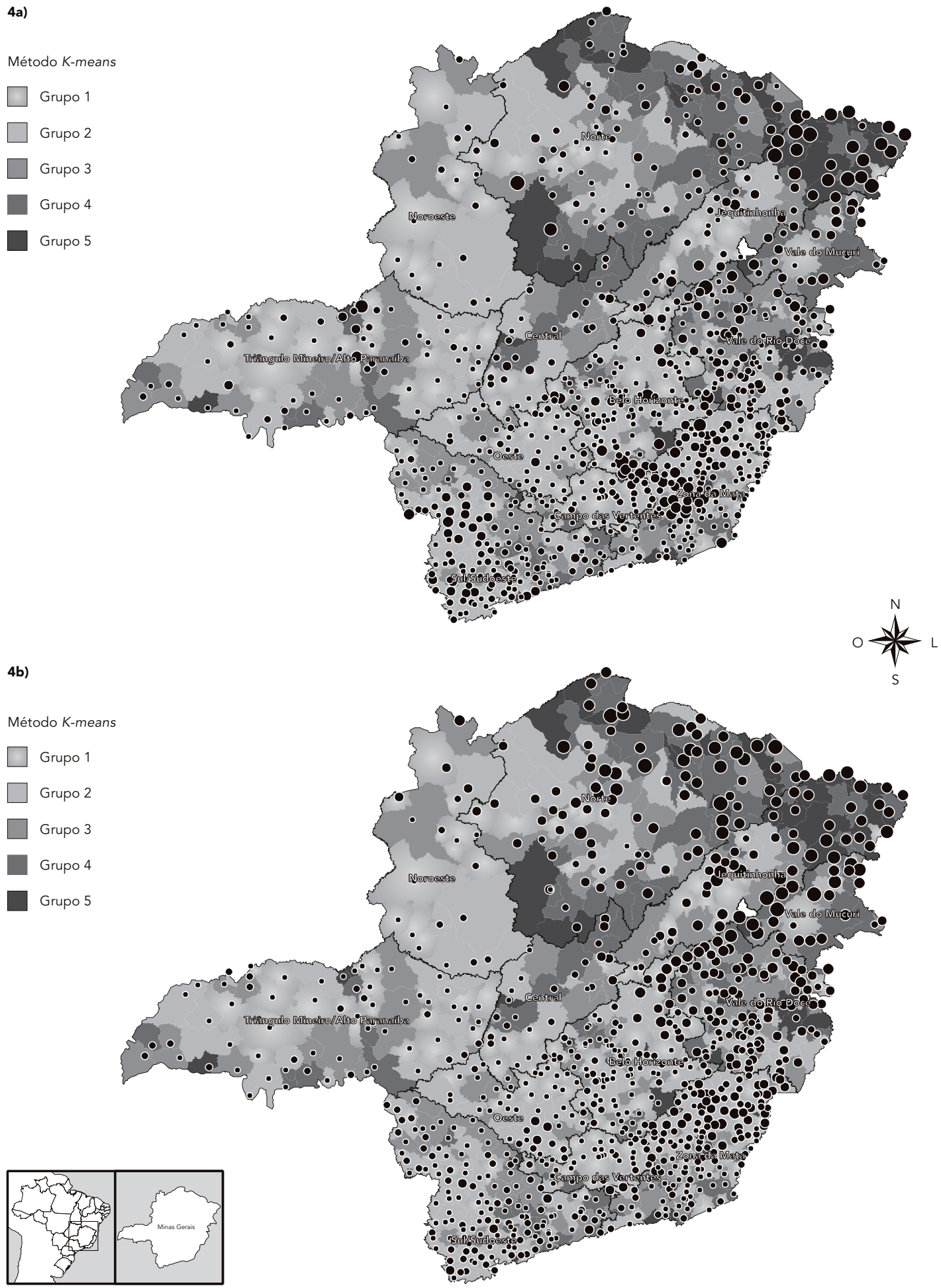


seu objetivo em cada município). Procurando-se correlacionar os grupos aos fatores determinantes para a ocorrência do câncer do colo do útero e ao desempenho das políticas públicas, apresenta-se na Figura $4 \mathrm{~b}$ a taxa de analfabetismo nas mulheres de 25 a 59 anos, ano 2000 e, portanto, a visualização da influência da terceira vertente no desempenho do programa (nível sócio-econômico das mulheres). As maiores proporções de mulheres que informaram não ter realizado o exame anteriormente (variação de $40 \%$ a $60 \%$ ) e as maiores taxas de mulheres não-alfabetizadas (variação de $22,98 \%$ a 53,56\%) foram encontradas nas mesorregiões Norte, Jequitinhonha, Vale do Mucuri e Vale do Rio Doce, coincidentes com as mesorregiões onde predominaram os grupos (4 e 5) com os maiores porcentuais de lâminas consideradas com adequabilidade "satisfatória, mas limitada por".

\section{Discussão}

Considerando as três vertentes que influenciam o Viva Mulher, viu-se que a variável percentual de lâminas consideradas com adequabilidade "satisfatória, mas limitada por”, que melhor discriminou os cinco grupos de municípios, pertencia à segunda vertente (capacidade operacional da rede de serviços). Algumas considerações podem ser feitas tendo em vista a baixa capacidade de discriminação das outras variáveis. Com relação à variável razão de exames realizados na população, este fato pode estar relacionado à segunda fase de intensificação do Viva Mulher, realizada em 2002, ao ampliar o acesso à coleta do material para o exame citopatológico, em esforço concentrado, homogeneizando a razão de exames realizados entre os municípios (variação da mediana de 0,24 a 0,28). Quanto às outras variáveis referentes aos tipos de lesão encontrados nas lâminas não foi observada uma variação significativa entre os grupos de municípios. Ressalte-se que essas variáveis são influenciadas não somente pela freqüência dos tipos de lesão na população, como também, pela existência de programa municipal com boa qualidade na coleta e preservação do material para o exame (Papanicolaou) e maior efetividade em buscar e acompanhar as mulheres durante o tratamento. Neste estudo, a ocorrência de lesões precursoras (NIC II e NIC III) e de câncer são observadas a partir do 3o quartil, sugerindo o quanto as mulheres residentes em municípios menos estruturados quanto à organização do programa podem estar desprovidas de recursos para a identificação e o acompanhamento do câncer e de suas lesões precursoras. Em que pese a possível existência da baixa prevalência das lesões para justificar a ocorrência, considerou-se a coincidência entre as dificuldades operacionais encontradas para a cobertura das mulheres e a coleta do material para exame.

Cabe considerar que as variáveis foram extraídas de um banco de dados oriundo de um sistema de pagamento de procedimentos realizados no SUS, não permitindo a mensuração de prevalência da doença na população. A inexistência de um identificador único para o usuário do SUS, pode fazer com que uma mesma mulher possa ser contada mais de uma vez, caso tenha realizado mais de um exame em um mesmo período. Tendo em vista as limitações atuais dos sistemas de informação vigentes no país, utilizam-se os porcentuais de lâminas com lesões e a razão de exames realizados na população como aproximações capazes de ajudar no planejamento de ações de controle da doença e de captação de mulheres (cobertura da população alvo). O pacto de atenção básica 21 , por exemplo, instrumento de monitoramento das ações de saúde em nível municipal, usa essa variável como parte do conjunto de indicadores referentes ao tópico saúde da mulher.

A representação espacial dos grupos também permitiu evidenciar alguns aspectos do Viva $\mathrm{Mu}-$ lher. Quanto ao aspecto da capacidade operacional, identificou-se, ao norte do Estado, uma maior concentração dos grupos 4 e 5, com pior qualidade na adequabilidade das lâminas e, do centro para o sul do Estado, uma concentração dos grupos 1 e 2, com qualidade melhor ou intermediária. No entanto, encontrou-se município com melhor qualidade ao norte, assim como município de pior qualidade ao sul. Isso pode significar que as ações desenvolvidas pelo programa variam conforme os esforços municipais, estando relacionadas com a gestão das políticas públicas locais, apesar das barreiras sociais e operacionais.

Ainda sob o aspecto operacional, a representação espacial permitiu supor que a segunda fase de intensificação, cujo esforço foi captar o maior número de mulheres em um intervalo de aproximadamente três meses em municípios com logísticas desiguais entre si, tenha sido responsável pela influência que teve a proporção de lâmina "satisfatória, mas limitada por" na análise de agrupamento. Basta observar que as subcategorias da lâmina "satisfatória, mas limitada por" tiveram comportamentos distintos, sendo as lâminas "dessecadas" encontradas mais ao norte e aquelas com "ausência de células endocervicais” mais ao sul. Isso aponta para a existência de problemas tanto na fixação (uso de fixador inadequado, quantidade insuficiente de fixador 
ou falta de fixação prévia) e transporte do material após a coleta (demora ou dificuldade no transporte da lâmina para o laboratório) ${ }^{3}$, quanto na coleta de material (células glandulares endocervicais existentes na zona de transformação do colo do útero) no local adequado 3,6 .

Considerando que a segunda fase de intensificação tenha contribuído para melhorar o acesso das mulheres à coleta do exame citopatológico em todo o Estado de Minas Gerais, é possível, por outro lado, que ela tenha agravado determinados tipos de problemas, principalmente aqueles referentes às questões operacionais. Tais problemas podem ser explicados em razão da natureza técnica do método utilizado no rastreamento (Papanicolaou), que envolve, por sua vez, uma grande necessidade de treinamento das equipes de saúde. Há, portanto, que se pesar a importância estratégica de uma campanha e a definição das táticas para implementá-la.

É importante destacar que a concentração de mulheres examinadas pela primeira vez nas mesmas regiões com taxas de analfabetismo mais altas, ao norte do estado, reafirmou o observado pelo inquérito populacional realizado pelo Ministério da Saúde, em 1994, onde o conhecimento das mulheres sobre o exame preventivo (Papanicolaou) foi diretamente proporcional ao grau de instrução e renda 22 .

Os achados deste estudo indicam que, no Estado de Minas Gerais, os fatores de processamento da lâmina (especialmente da sua coleta) são mais críticos do que aqueles relacionados ao acesso das mulheres ao programa (razão de exames realizados na população alvo). Para o tipo de problema encontrado, foi primordial a existência de uma requisição de exame citopatológico do colo do útero informatizada, discriminando em campos distintos as subcategorias para os três tipos de adequabilidade do material coletado: "satisfatória"; "satisfatória, mas limitada por", com sete subcategorias; e "insatisfatória", com oito subcategorias. Há que se refletir, portanto, quanto às modificações previstas na Nomenclatura Brasileira para Laudos Citopatológicos Cervicais e Condutas Clínicas Preconizadas 23 , similar ao sistema Bethesda - 2001, onde a adequabilidade da amostra passa a ter duas classificações: "satisfatória” e "insatisfatória”, além da distinção (obrigatória) sobre os epitélios representados na amostra (escamoso, glandular e metaplásico). Dessa forma, ainda que previstos sete campos distintos para os diferentes tipos da classificação "insatisfatória", perde-se, no entanto, a possibilidade de coletar dados referentes aos fatores operacionais para a obtenção de uma amostra "satisfatória" (células em quantidade representativa, bem distribuídas, fixadas e coradas). Neste estudo, a variável fundamental na classificação dos municípios foi exatamente a "satisfatória, mas limitada por”, que não está contemplada na nova nomenclatura. Cabe considerar então, se as duas categorias de adequabilidade da lâmina serão suficientes para discriminar os municípios quanto aos problemas relacionados aos fatores operacionais, fundamentais para o direcionamento da capacitação das equipes de saúde.

A gestão estadual da saúde tem, dada a realidade desigual dos municípios, um importante papel fomentador na superação das desigualdades. Como evidenciado, há que ser realizado um grande esforço para treinamento de médicos e enfermeiras na coleta e preservação do material para o exame citopatológico (Papanicolaou), priorizando determinados grupos de municípios. A centralização de laboratórios evidenciada no estudo pareceu-nos excessiva para a operacionalização do programa, especialmente ao norte do Estado. Há que se pensar nas dimensões territoriais de Minas Gerais, dificultando o transporte rápido das lâminas para o laboratório, fato que parece ter influenciado a preservação do material coletado na mesorregião norte, que conta para a leitura das lâminas com apenas três municípios: Brasília de Minas (8.476), Francisco Sá (9.517) e Montes Claros (46.112). Assim, é importante rever a centralização da prestação de serviços laboratoriais, tornando-a mais presente no estado.

A incorporação de metodologia de análise multivariada de dados tendo como objeto a classificação dos municípios para diferentes dimensões ou vertentes pode facilitar, não somente, o planejamento das Secretarias de Saúde (Estadual e Municipal) na resolução de problemas operacionais do programa, como também, facilitar a elaboração de estratégias voltadas para o aumento da captação de mulheres para o rastreamento e a identificação do câncer e suas lesões precursoras. 


\section{Resumo}

Neste trabalho estudou-se o perfil de produção do exame citopatológico (Papanicolaou) realizado pelo Programa de Controle do Câncer do Colo do Útero, no ano de 2002, em mulheres na faixa etária de 25 a 59 anos, residentes em 850 municípios do Estado de Minas Gerais, Brasil. A análise de agrupamento foi utilizada para classificar os municípios quanto à quantidade de exames citopatológicos realizados na população, às alterações encontradas nos exames e à adequabilidade das lâminas. A variável "lâmina satisfatória, mas limitada por" foi o principal fator de discriminação dos municípios em cinco grupos. A representação espacial desses grupos apontou uma situação mais crítica nas mesorregiões Norte, Jequitinhonha, Vale do Mucuri e Vale do Rio Doce; e problemas operacionais decorrentes da coleta inadequada de material, sendo observadas mais lâminas "dessecadas" ao norte do Estado e mais lâminas com "ausência de células endocervicais" do centro para o sul. A metodologia adotada permitiu identificar grupos de municípios com problemas na execução do programa quanto à qualidade da coleta de material, à fixação e à leitura da lâmina para o exame citopatológico.

Neoplasias do Colo do Útero; Programas de Rastreamento; Técnicas Citológicas

\section{Colaboradores}

T. M. P. Feitosa contribuiu na construção e manipulação da base de dados, na análise de agrupamento para classificação dos municípios e na análise e discussão dos resultados. R. T. Almeida contribuiu na validação da classificação dos municípios e na análise e discussão dos resultados. Ambas contribuíram na redação do texto.

\section{Agradecimentos}

Os autores agradecem a Ernani Bento Bandarra, José do Vale Pinheiro Feitosa e Lucília Zardo por suas valiosas contribuições para a elaboração deste artigo, assim como ao Instituto Nacional de Câncer pelo apoio à realização deste trabalho.

\section{Referências}

1. World Health Organization. Manual on the prevention and control of common cancers. Geneva: World Health Organization; 1998. (Westerns Pacific Series, 20).

2. World Health Organization. National cancer control programmes: policies and managerial guidelines. 2nd Ed. Geneva: World Health Organization; 2002.

3. World Health Organization. Cervical cancer screening in developing countries: report of a WHO consultation. Geneva: World Health Organization; 2002.

4. Pinotti JA, Zeferino LC. Programa de controle de câncer cérvico uterino. Campinas: Editora da Unicamp; 1987.

5. Departamento de Informação e Informática do SUS. Sistema de informações ambulatoriais - SIA/ SUS. http://tabnet.datasus.gov.br/cgi/sia/padescr. htm (acessado em 09/Out/2003).

6. Instituto Nacional de Câncer. Falando sobre câncer do colo do útero. http://www.inca.gov.br (acessado em 27/Nov/2003).
7. Instituto Nacional de Câncer. Inquérito domiciliar sobre comportamentos de risco e morbidade referida de doenças e agravos não transmissíveis. Rio de Janeiro: Instituto Nacional de Câncer; 2004.

8. Instituto Nacional de Câncer. Viva mulher. Câncer do colo do útero: informações técnico-gerenciais e ações desenvolvidas. Rio de Janeiro: Instituto Nacional de Câncer; 2002.

9. Caetano R, Caetano CMM. Custo-efetividade no rastreamento do câncer cérvico-uterino no Brasil: um estudo exploratório. http://www.inca.gov.br/ inca/Arquivos/HPV/relatorio\%20do\%20estudo\%2 0HPV.pdf (acessado em 06/Mar/2006).

10. Instituto Nacional de Câncer. Estimativa 2006: in cidência de câncer no Brasil. http://www.inca.gov. br/estimativa/2006/ (acessado em 06/Mar/2006).

11. Guerra MR, Gallo CVM, Mendonça GAS. Risco de câncer no Brasil: tendências e estudos epidemiológicos mais recentes. Rev Bras Cancerol 2005; 51:227-34

12. Secretaria de Vigilância em Saúde. Saúde Brasil 2004: uma análise da situação de saúde. Brasília: Ministério da Saúde; 2004. 
13. Ministério da Saúde. Normas e recomendações do Instituto Nacional de Câncer: recomendações básicas para o controle do câncer do colo do útero no Brasil. Rev Bras Cancerol 2000; 46:23-33.

14. Ministério da Saúde. Condutas do Instituto Nacional de Câncer: câncer do colo do útero. Neoplasia intra-epitelial cervical - NIC. Rev Bras Cancerol 2000; 46:355-7.

15. Departamento de Informação e Informática do SUS. Sistema de informações do câncer da mulher. http://www.datasus.gov.br (acessado em 09/ Out/2003).

16. Instituto Brasileiro de Geografia e Estatística. Projeção populacional da população residente -2000 : Brasil, unidades da federação e municípios. http:// www.ibge.gov.br (acessado em 09/Out/2003).

17. Aldenderfer MS, Blashfield RK. Cluster analysis. Newbury Park: Sage University Papers; 1990.

18. Dilts D, Khamalah J, Plotkin A. Using cluster analysis for medical resource decision making. Med Decis Making 1995; 15:333-47.
19. Johnson RA, Wicherin DW. Applied multivariate statistical analysis. New Jersey: Prentice-Hall; 1992.

20. Pereira JCR. Análise de dados qualitativos: estratégias metodológicas para as ciências da saúde, humanas e sociais. 2a Ed. São Paulo: Edusp; 1999.

21. Ministério da Saúde. Portaria no. 21, de 5 de janeiro de 2005. Aprova os indicadores da atenção básica - 2005. http://tabnet.datasus.gov.br/cgi/ siab/pacto2005/portaria21.pdf (acessado em 03/ Nov/2005).

22. Lopes ER, Rebelo MS, Abreu E, Costa-Silva VL, Eisenberg ALA, Lavor MF. Comportamento da população brasileira feminina em relação ao câncer cérvico-uterino. J Bras Ginecol 1995; 105:505-16.

23. Instituto Nacional de Câncer. Nomenclatura brasileira para laudos citopatológicos cervicais e condutas clínicas preconizadas. Rio de Janeiro: Instituto Nacional de Câncer; 2003.

Recebido em 22/Ago/2005

Versão final reapresentada em 05/Abr/2006

Aprovado em 03/Jul/2006 